\title{
RATIONAL APPROXIMATIONS TO THE DILOGARITHM
}

\author{
MASAYOSHI HATA
}

\begin{abstract}
The irrationality proof of the values of the dilogarithmic function $L_{2}(z)$ at rational points $z=1 / k$ for every integer $k \in(-\infty,-5] \cup[7, \infty)$ is given. To show this we develop the method of Padé-type approximations using Legendre-type polynomials, which also derives good irrationality measures of $L_{2}(1 / k)$. Moreover, the linear independence over $\mathbf{Q}$ of the numbers 1 , $\log (1-1 / k)$, and $L_{2}(1 / k)$ is also obtained for each integer $k \in(-\infty,-5] \cup$ $[7, \infty)$.
\end{abstract}

\section{INTRODUCTION}

This paper is devoted to studying rational approximations to the values of the dilogarithmic function

$$
L_{2}(z)=-\int_{0}^{z} \frac{\log (1-t)}{t} d t=\sum_{n=1}^{\infty} \frac{z^{n}}{n^{2}}
$$

at rational points $z=1 / k$ for nonzero integers $k$. The function $L_{2}(z)$ was studied over two hundred years ago by Euler, Landen, and others. The special values of $L_{2}(z)$ at some algebraic points and several functional relations satisfied by $L_{2}(z)$ are known (see, for example, Lewin's book [9, Chapter 1]). $L_{2}(z)$ is holomorphic in the complex plane with a branch cut $z=1$ to $\infty$.

Concerning arithmetical properties of the values of dilogarithms at rational points, Maier [10] has shown the irrationality of $L_{2}(1 / k)$ for any integer $k \geq 2^{8} e^{2}$. This result was remarkably improved by Chudnovsky [2, 3], who announced that $L_{2}(1 / k)$ is irrational for any integer $k \geq 14$. He also pointed out that in the $G$-function approach (ineffective method) $L_{2}(1 / k)$ becomes irrational only for $k \geq e^{127}$. Recently the author [8] studied Padé-type approximations to the dilogarithm by using the Legendre-type polynomials with integral coefficients

$$
\begin{aligned}
A_{n}(x) & =\frac{1}{n_{1} ! n_{2} !}\left(x^{n_{2}}\left(x^{n_{1}}(1-x)^{n}\right)^{\left(n_{1}\right)}\right)^{\left(n_{2}\right)} \\
& =\sum_{j=0}^{n}(-1)^{j}\left(\begin{array}{c}
n \\
j
\end{array}\right)\left(\begin{array}{c}
n_{1}+j \\
n_{1}
\end{array}\right)\left(\begin{array}{c}
n_{2}+j \\
n_{2}
\end{array}\right) x^{j},
\end{aligned}
$$

Received by the editors December 17, 1990.

1980 Mathematics Subject Classification (1985 Revision). Primary 11J82; Secondary 11 J72.

Key words and phrases. Dilogarithm, irrationality measure, Padé approximation. 
where $n_{1}=[n / 2]$ and $n_{2}=[(n+1) / 2]$. Then he showed that $L_{2}(1 / k)$ is irrational for any integer $k \in(-\infty,-8] \cup[12, \infty)$.

Modifying the polynomials (1.1), we now introduce the following polynomials with integral coefficients:

$$
\begin{aligned}
B_{l, m, n}(x) & =\frac{1}{l ! m !}\left(x^{l}\left(x^{m+n}(1-x)^{l+m}\right)^{(m)}\right)^{(l)} \\
& =\sum_{j=0}^{l+m}(-1)^{j}\left(\begin{array}{c}
l+m \\
j
\end{array}\right)\left(\begin{array}{c}
j+m+n \\
m
\end{array}\right)\left(\begin{array}{c}
j+l+n \\
l
\end{array}\right) x^{j+n},
\end{aligned}
$$

where $l, m(l \geq m)$, and $n$ are nonnegative integers. The importance of these polynomials lies in the fact that the greatest common divisor of the coefficients is fairly large. Then the polynomials (1.2) enable us to obtain the following

Theorem 1.1. $L_{2}(1 / k)$ is irrational for any integer $k \in(-\infty,-5] \cup[7, \infty)$.

The proof of Theorem 1.1 will be given in $\S 6$.

Our method also provides good irrationality measures of $L_{2}(1 / k)$. For example,

$$
\left|L_{2}\left(\frac{1}{14}\right)-\frac{p}{q}\right| \geq q^{-15.275}
$$

for any sufficiently large integer $q$, which fairly improves Chudnovsky's result: $\mu(14) \simeq 300$ [3]. For some numerical results on the irrationality measures of $L_{2}(1 / k)$, see Tables 1 and 2 in $\S 6$.

Although our method cannot be applied to the case $k=1$, there are several results concerning rational approximations to the value $L_{2}(1)=\pi^{2} / 6$. (See van der Poorten [11], Beukers [1], Chudnovsky and Chudnovsky [4], Dvornicich and Viola [5], Rukhadze [12], and the author's paper [7].)

Analogously to [8] we can also show that the numbers $1, \log (1-1 / k)$, and $L_{2}(1 / k)$ are linearly independent over $\mathbf{Q}$ for any integer $k \in(-\infty,-5] \cup$ $[7, \infty)$.

In $\S \S 2$ and 3, we will investigate some fundamental properties of the polynomials (1.2). Then we will construct explicitly our Padé-type approximations to the dilogarithm $L_{2}(z)$ in $\S 4$. In $\S 5$ the asymptotic behaviors of the denominators of the approximate rational numbers and of the remainder terms will be given. Our main results on the irrationality measures of $L_{2}(1 / k)$ will be proved in the last section.

\section{Preliminaries}

In this section we investigate some fundamental properties on the polynomials $B_{l, m, n}(x)$. First, we have the following

Lemma 2.1. Let $l, m, n$, and $n^{\prime}$ be nonnegative integers satisfying $l \geq m$, and suppose that $P(x)$ is a real polynomial of degree less than $l+m+n+n^{\prime}$ satisfying the following properties:

(1) $\int_{0}^{1} x^{j} P(x) d x=0$ for $0 \leq j<l$;

(2) $\int_{0}^{1} x^{j} P(x) \log x d x=0$ for $0 \leq j<m$;

(3) $P(x)=O\left(x^{n}\right)$ as $x \rightarrow 0$ and $P(x)=O\left((1-x)^{n^{\prime}}\right)$ as $x \rightarrow 1$.

Then $P(x)$ must be identically zero. 
Proof. Put

$$
\widetilde{P}(x)=\underbrace{\int_{0}^{x} \int_{0}^{t_{m}} \cdots \int_{0}^{t_{2}}}_{m \text { times }} P\left(t_{1}\right) d t_{1} d t_{2} \cdots d t_{m} .
$$

Then it follows from (1) and (3) that $\widetilde{P}(x)=x^{m+n}(1-x)^{m+n^{\prime}} Q(x)$ for some real polynomial $Q(x)$ of degree less than $l$, and that $\int_{0}^{1} x^{j} \widetilde{P}(x) d x=0$ for $0 \leq j<l-m$. Moreover, this equality holds also for every $-m \leq j \leq-1$ from (2). Thus

$$
\int_{0}^{1} x^{j+n}(1-x)^{m+n^{\prime}} Q(x) d x=0
$$

for $0 \leq j<l$; in particular,

$$
\int_{0}^{1} x^{n}(1-x)^{m+n^{\prime}} Q^{2}(x) d x=0 .
$$

Therefore we have $Q(x) \equiv 0$; hence $P(x) \equiv 0$, as required.

It is easily verified that the polynomial

$$
B_{l, m, n, n^{\prime}}(x)=\frac{1}{l ! m !}\left(x^{l}\left(x^{m+n}(1-x)^{l+m+n^{\prime}}\right)^{(m)}\right)^{(l)}
$$

of degree $l+m+n+n^{\prime}$ satisfies the conditions (1), (2), and (3) of Lemma 2.1 if $l \geq m$. Therefore these conditions determine the polynomial (2.1) uniquely up to a constant multiple.

Next we study the distribution of zero points of $B_{l, m, n, n^{\prime}}(x)$.

Lemma 2.2. If $l \geq m$, then $B_{l, m, n, n^{\prime}}(x)$ has $l+m$ simple zeros in the open unit interval $(0,1)$. Hence all zero points of $B_{l, m, n, n^{\prime}}(x)$ lie on the closed unit interval $[0,1]$.

Proof. We can assume that $l \geq 1$. Let $N(U)$ be the number of zero points of $U(x)$ with odd order lying on $(0,1)$ for any real polynomial $U(x) \not \equiv 0$. Put

$$
\widetilde{B}(x)=\underbrace{\int_{0}^{x} \int_{0}^{t_{m}} \cdots \int_{0}^{t_{2}}}_{m \text { times }} B_{l, m, n, n^{\prime}}\left(t_{1}\right) d t_{1} d t_{2} \cdots d t_{m}
$$

Then, using the same method as in the previous proof, $\int_{0}^{1} x^{j} B^{*}(x) d x=0$ for $0 \leq j<l$, where $B^{*}(x) \equiv x^{-m} \widetilde{B}(x)$ is a real polynomial of degree $l+m+$ $n+n^{\prime} \geq 1$; hence $N(\widetilde{B})=N\left(B^{*}\right) \geq l$ by the standard argument. Therefore we have $N\left(B_{l, m, n, n^{\prime}}\right)=N\left(\widetilde{B}^{(m)}\right) \geq N\left(\widetilde{B}^{(m-1)}\right)+1 \geq \cdots \geq N(\widetilde{B})+m \geq l+m$. Since $x=0$ and $x=1$ are zero points of $B_{l, m, n, n^{\prime}}(x)$ of order $n$ and $n^{\prime}$ respectively, $B_{l, m, n, n^{\prime}}(x)$ must have $l+m$ simple zeros in $(0,1)$. This completes the proof.

In particular, $B_{l, m, n, n^{\prime}}(z) \neq 0$ for any $z \in \mathbf{C}-[0,1]$.

We now study further properties of the polynomials $B_{l, m, n}(x) \equiv B_{l, m, n, 0}(x)$. Let $\lambda>1$ be an arbitrarily fixed real number. From now on we restrict ourselves to the following case:

$$
m \equiv m(N)=\left[\frac{1}{2}\left(N-\left[\frac{3 N}{2 \lambda+1}\right]\right)\right], \quad n \equiv n(N)=\left[\frac{1}{3}\left[\frac{3 N}{2 \lambda+1}\right]\right]
$$


and $l \equiv l(N)=N-m(N)-n(N)$ for any nonnegative integer $N$. Note that $l(N), m(N)$, and $n(N)$ are monotone nondecreasing functions on $N$ and that $l(N) \geq m(N)+2 n(N)$ for all $N \geq 0$. For brevity, put

$$
P_{\lambda, N}(x) \equiv B_{l(N), m(N), n(N)}(x) .
$$

Clearly $P_{\lambda, N}(x)$ is a polynomial of degree $N$ with integral coefficients. Then the system of polynomials $\left\{P_{\lambda, N}(x)\right\}_{N \geq 0}$ satisfies the following recurrence formula.

Lemma 2.3. Let $K=8+[11 /(\lambda-1)]$. Then, for any $N \geq K$,

$$
P_{\lambda, N+1}(z) \equiv\left(a_{\lambda, N} z+b_{\lambda, N}\right) P_{\lambda, N}(z)+\sum_{j=1}^{K} c_{j, \lambda, N} P_{\lambda, N-j}(z),
$$

where $a_{\lambda, N}, b_{\lambda, N}$, and $c_{j, \lambda, N}(1 \leq j \leq K)$ are rational constants depending only on $\lambda$ and $N$. Moreover, the systems of the functions

$$
I_{\lambda, N}(z)=\int_{0}^{1} \frac{P_{\lambda, N}(t)}{t-z} d t \text { and } J_{\lambda, N}(z)=\int_{0}^{1} \frac{P_{\lambda, N}(t)}{t-z} \log t d t
$$

also satisfy the same linear recurrence formula $(2.3)$ for any $z \in \mathbf{C}-[0,1]$.

Proof. Put

$$
\begin{aligned}
P^{*}(z)= & P_{\lambda, N+1}(z)-\left(a_{\lambda, N} z+b_{\lambda, N}\right) P_{\lambda, N}(z) \\
& -\sum_{j=1}^{K} c_{j, \lambda, N} P_{\lambda, N-j}(z),
\end{aligned}
$$

where $a_{\lambda, N}, b_{\lambda, N}$, and $c_{j, \lambda, N}(1 \leq j \leq K)$ are rational numbers chosen so that the degree of $P^{*}(z)$ is less than $N-K$. Clearly we have $\int_{0}^{1} x^{j} P^{*}(x) d x=0$ for $0 \leq j<l^{*}$, where

$$
l^{*}=\min \{l(N+1), l(N)-1, l(N-1), \ldots, l(N-K)\} .
$$

Since $K \geq 8$, it is easily seen that $l(N)-1 \geq l(N-K)$; hence $l^{*}=l(N-K)$. Similarly, $\int_{0}^{1} x^{j} P^{*}(x) \log x d x=0$ for $0 \leq j<m^{*}$, where

$$
m^{*}=\min \{m(N+1), m(N)-1, m(N-1), \ldots, m(N-K)\}=m(N-K)
$$

since $K=8+[11 /(\lambda-1)]$. Moreover, $P^{*}(x)=O\left(\dot{x}^{n^{*}}\right)$ as $x \rightarrow 0$, where

$$
n^{*}=\min \{n(N+1), n(N), n(N-1), \ldots, n(N-K)\}=n(N-K) .
$$

Thus, from Lemma 2.1, $P^{*}(z) \equiv 0$ since $N-K=l(N-K)+m(N-K)+$ $n(N-K)$, as required.

Next we have

$$
\begin{gathered}
I_{\lambda, N+1}(z)-\left(a_{\lambda, N} z+b_{\lambda, N}\right) I_{\lambda, N}(z)-\sum_{j=1}^{K} c_{j, \lambda, N} I_{\lambda, N-j}(z) \\
=a_{\lambda, N} \int_{0}^{1} P_{\lambda, N}(x) d x=0
\end{gathered}
$$

since $l(N) \geq l(K) \geq 3$. The same equality holds for the system $\left\{J_{\lambda, N}(z)\right\}_{N \geq 0}$ since $m(N) \geq m(K) \geq 3$. This completes the proof.

Since the recurrence formula (2.3) is linear, we have immediately 
Corollary 2.4. Suppose that there exist an integer $N_{0} \geq 0$, a complex number $z_{0} \in \mathbf{C}-[0,1]$, and complex constants $u_{0}, v_{0}$, and $w_{0}$ satisfying

$$
u_{0} P_{\lambda, j}\left(z_{0}\right)+v_{0} I_{\lambda, j}\left(z_{0}\right)+w_{0} J_{\lambda, j}\left(z_{0}\right)=0
$$

for $N_{0} \leq j \leq N_{0}+K$. Then this equality holds for all $j \geq N_{0}$.

We also need the following lemma, which will play an important role in the proof of the irrationality of $L_{2}(1 / k)$ and even in the proof of the linear independence of the numbers $1, \log (1-1 / k)$, and $L_{2}(1 / k)$.

Lemma 2.5. Let $M$ be a nonnegative integer. Suppose that $g(x) \in L^{1}(0,1)$ satisfies

$$
\int_{0}^{1} P_{\lambda, j}(x) g(x) d x=0
$$

for all $j \geq M$. Then the function $g(x)$ can be expressed in the form

$$
g(x)=U(x)+V(x) \log x
$$

almost everywhere for some polynomials $U(x)$ and $V(x)$ of degrees less than $l(M)$ and $m(M)$ respectively.

Proof. We put $l=l(M), m=m(M)$, and $n=n(M)$ for brevity. For any vector $\mathbf{v}=\left(a_{0}, a_{1}, \ldots, a_{l-1}, b_{0}, \ldots, b_{m-1}\right) \in \mathbf{R}^{l+m}$, we define

$$
\phi_{\mathbf{v}}(x)=\sum_{j=0}^{l-1} a_{j} x^{j}+\sum_{j=0}^{m-1} b_{j} x^{j} \log x ;
$$

so the above lemma states that $g(x)=\phi_{\mathbf{v}}(x)$ almost everywhere for some vector $\mathbf{v}$.

We first show that the mapping $\Phi: \mathbf{R}^{l+m} \rightarrow \mathbf{R}^{l+m}$ defined by

$$
\Phi(\mathbf{v})=\left(\int_{0}^{1} x^{n} \phi_{\mathbf{v}}(x) d x, \int_{0}^{1} x^{n+1} \phi_{\mathbf{v}}(x) d x, \ldots, \int_{0}^{1} x^{M-1} \phi_{\mathbf{v}}(x) d x\right)
$$

is a homeomorphism. Let $X_{\Phi}$ be the corresponding matrix representation of $\Phi$. To show that the determinant of $X_{\Phi}$ does not vanish, it suffices to show that $X_{\Phi}$ has no zero-eigenvalues. Suppose, on the contrary, that $\Phi(\mathbf{v})=0$ for some $\mathbf{v} \neq \mathbf{0}$; that is,

$$
\int_{0}^{1} x^{j+n} \phi_{\mathbf{v}}(x) d x=0
$$

for any $0 \leq j<l+m$. Put

$$
G(x)=\underbrace{\int_{0}^{1-x} \int_{0}^{t_{l+m}} \cdots \int_{0}^{t_{2}}}_{l+m \text { times }} t_{1}^{n} \phi_{\mathbf{v}}\left(t_{1}\right) d t_{1} d t_{2} \cdots d t_{l+m} .
$$

Then it follows from $(2.4)$ that $G^{(j)}(0)=0$ for any $0 \leq j<l+m$. On the other hand, since $l \geq m$, it is easily seen that

$$
G(z)=(1-z)^{M}\left(U_{0}(z)+V_{0}(z) \log (1-z)\right)
$$

for some polynomials $U_{0}(z)$ and $V_{0}(z)$ of degrees less than $l$ and $m$ respectively. Since $G(z)$ is an analytic function in a neighborhood of the origin, we 


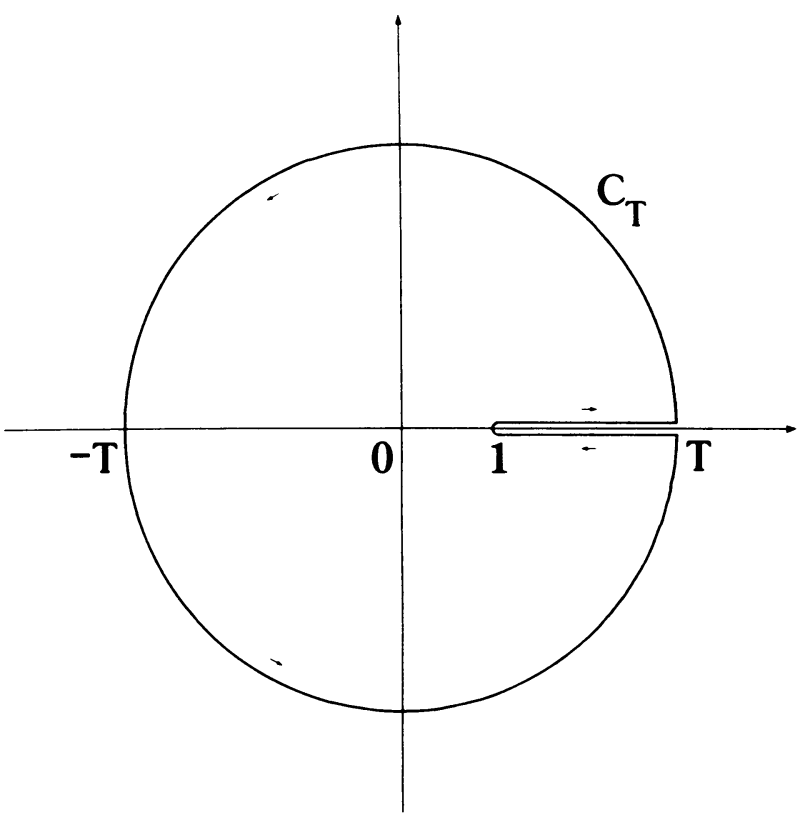

FIGURE 1. The contour $C_{T}$

have $U_{0}(z)+V_{0}(z) \log (1-z)=O\left(z^{l+m}\right)$ as $z \rightarrow 0$. Then we have $m \geq 1$; for otherwise, $U_{0} \equiv V_{0} \equiv 0$, contrary to $\phi_{\mathbf{v}} \not \equiv 0$. Since the function $V_{0}(z) \log (1-z)$ does not contain the terms of $z^{j+l}$ for $0 \leq j<m$ in its Taylor expansion at $z=0$, we obtain

$$
\frac{1}{2 \pi i} \int_{C} \frac{V_{0}(z) \log (1-z)}{z^{j+l+1}} d z=0
$$

for any $0 \leq j<m$, where $C$ is a sufficiently small circle centered at the origin. In particular, since $z^{m-1} V_{0}(1 / z)$ is a polynomial of degree less than $m$, we have

$$
\frac{1}{2 \pi i} \int_{C} \frac{V_{0}^{2}(z) \log (1-z)}{z^{l+m}} d z=0 .
$$

The integrand of the above integral is clearly analytic in the complex plane with a branch cut from $z=1$ to $\infty$. So we can change the contour $C$ to the new contour $C_{T}$ consisting of the circle centered at the origin with radius $T>1$ and two segments joining $z=1$ and $z=T$ (see Figure 1). Letting $T \rightarrow \infty$, we thus obtain

$$
\int_{1}^{\infty} \frac{V_{0}^{2}(t)}{t^{l+m}} d t=0
$$

therefore $V_{0}(z) \equiv 0$ and hence $U_{0}(z) \equiv 0$. This contradiction implies that $\Phi$ is a homeomorphism, as required.

Put $g_{\mathbf{v}}(x)=g(x)-\phi_{\mathbf{v}}(x) \in L^{1}(0,1)$ for brevity. Since $\Phi$ is a homeomorphism, there exists a unique vector $\mathbf{w} \in \mathbf{R}^{l+m}$ such that

$$
\int_{0}^{1} x^{j+n} g_{\mathbf{w}}(x) d x=0
$$

for $0 \leq j<l+m$. On the other hand, since $\int_{0}^{1} P_{\lambda, N}(x) \phi_{\mathbf{w}}(x) d x=0$ for all 
$N \geq M$, we have

$$
\int_{0}^{1} P_{\lambda, N}(x) g_{\mathbf{w}}(x) d x=0
$$

for all $N \geq M$. Now $x^{-n} P_{\lambda, N}(x)$ is a polynomial of degree $N-n \geq l+m$. Hence it follows from (2.5) and (2.6) that the integrable function $x^{n} g_{\mathbf{w}}(x)$ is orthogonal to all polynomials. By the standard argument, we thus have $g_{w}(x)=$ 0 almost everywhere. This completes the proof.

Combining Corollary 2.4 and the above lemma, we have

Corollary 2.6. Suppose that there exist an integer $N_{0} \geq 0$, a complex number $z_{0} \in \mathbf{C}-[0,1]$, and complex constants $v_{0}$ and $w_{0}$ satisfying

$$
v_{0} I_{\lambda, j}\left(z_{0}\right)+w_{0} J_{\lambda, j}\left(z_{0}\right)=0
$$

for $N_{0} \leq j \leq N_{0}+K$. Then $v_{0}=w_{0}=0$.

Proof. By Corollary 2.4, (2.7) holds for all $j \geq N_{0}$; in other words,

$$
\int_{0}^{1} P_{\lambda, j}(x) g(x) d x=0
$$

for all $j \geq N_{0}$, where

$$
g(x)=\frac{v_{0}+w_{0} \log x}{x-z_{0}} \in L^{1}(0,1) .
$$

Thus, by Lemma 2.5 , there exist some polynomials $U(x)$ and $V(x)$ such that $g(x)=U(x)+V(x) \log x$ for any $0<x<1$. This implies that

$$
\tilde{U}(x)+\tilde{V}(x) \log x \equiv 0
$$

where $\tilde{U}(x)=v_{0}-\left(x-z_{0}\right) U(x)$ and $\tilde{V}(x)=w_{0}-\left(x-z_{0}\right) V(x)$. Then it is easily seen that this occurs if and only if $\tilde{U}(x) \equiv \widetilde{V}(x) \equiv 0$; hence $U(x) \equiv V(x) \equiv 0$ and $v_{0}=w_{0}=0$, as required.

\section{ARITHMETICAL PROPERTY OF THE COEFFICIENTS}

In this section we study the asymptotic behavior of common divisors of the coefficients of $P_{\lambda, N}(x)$ as $N \rightarrow \infty$. The main result (Lemma 3.1 below) in this section is an arithmetical lemma, which will be proved by using the prime number theorem.

We denote by $(x, y)$ the usual Cartesian coordinates in $\mathbf{R}^{2}$. Then define the "characteristic set" $D=D_{1} \cup D_{2} \cup D_{3}$, where

$$
\begin{aligned}
& D_{1}=\left\{(x, y) ; y>\frac{1}{2}, x+y<1, \text { and } 2 x+y>1\right\}, \\
& D_{2}=\left\{(x, y) ; y>\frac{1}{2}, y<x, \text { and } x+2 y<2\right\}, \\
& D_{3}=\{(x, y) ; y>0, y<x, \text { and } x+2 y<1\} .
\end{aligned}
$$

Each set $D_{j}$ is an open triangle contained in $[0,1] \times[0,1]$ (see Figure 2). We also denote by $\{x\}$ the fractional part of $x$. For any real number $\lambda>1$ and any subset $W \subset[0,1] \times[0,1]$, we define

$$
E_{\lambda}(W)=\{x>0 ;(\{x\},\{\lambda x\}) \in W\} .
$$




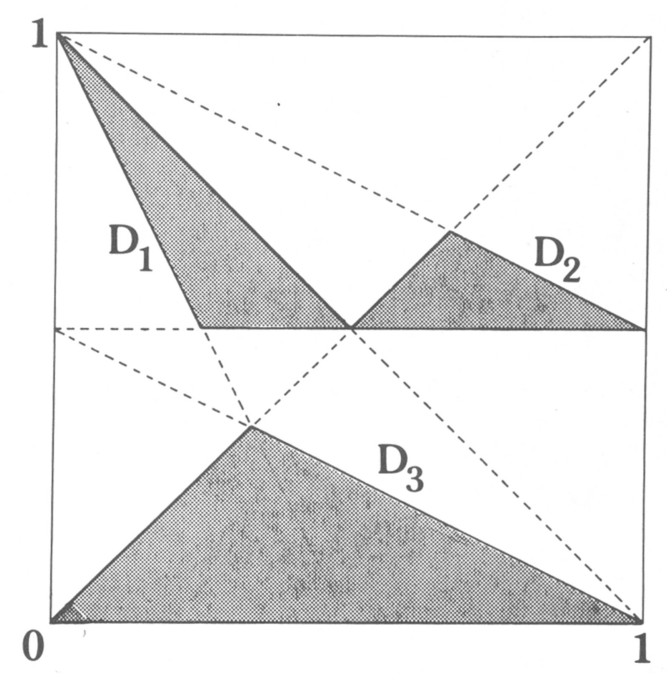

Figure 2. The characteristic set $D=D_{1} \cup D_{2} \cup D_{3}$

Clearly the set $E_{\lambda}(D)$ is a countable union of open intervals. We then define $e(\lambda)=\int_{E_{\lambda}(D)} d x / x^{2}$. Our result can now be stated as follows.

Lemma 3.1. Let $\lambda>1$ be a real parameter. Then there exists a common divisor $d_{\lambda}(N)$ of the following integers:

$$
\left(\begin{array}{c}
l(N)+m(N) \\
j
\end{array}\right)\left(\begin{array}{c}
j+m(N)+n(N) \\
m(N)
\end{array}\right)\left(\begin{array}{c}
j+l(N)+n(N) \\
l(N)
\end{array}\right)
$$

for $0 \leq j \leq l(N)+m(N)$ satisfying

$$
\lim _{N \rightarrow \infty} \frac{1}{N} \log d_{\lambda}(N)=\frac{e(\lambda)}{2 \lambda+1} .
$$

Proof. Put $\delta_{N}=3 / \sqrt{N}$ for brevity. For any sufficiently large $N$, we first define

$$
D_{j}^{(N)}=\left\{(x, y) \in \mathbf{R}^{2} ; \operatorname{dist}\left((x, y), \mathbf{R}^{2}-D_{j}\right)>\sqrt{2} \delta_{N}\right\}
$$

for $1 \leq j \leq 3$ and put $D^{(N)}=D_{1}^{(N)} \cup D_{2}^{(N)} \cup D_{3}^{(N)}$. Clearly $E_{\lambda}\left(D^{(N)}\right)$ is a countable union of open intervals and $\left\{E_{\lambda}\left(D^{(N)}\right)\right\}$ is a monotone increasing sequence of open sets such that $\bigcup E_{\lambda}\left(D^{(N)}\right)=E_{\lambda}(D)$. Note that $\operatorname{dist}\left(D_{i}^{(N)}, D_{j}^{(N)}\right) \geq$ $2 \sqrt{2} \delta_{N}$ for any $i \neq j$. Now let $H_{N}$ be the set of all prime numbers $p>\sqrt{2 N}$ satisfying $N /(2 \lambda+1) p \in E_{\lambda}\left(D^{(N)}\right)$. Then we define

$$
d_{\lambda}(N)=\prod_{p \in H_{N}} p
$$

We now show that each $p \in H_{N}$ divides all the coefficients of the polynomial $P_{\lambda, N}(x)$. To see this, for an arbitrarily fixed $j \in[0, l(N)+m(N)]$, let $\omega, \eta$, $\eta^{\prime}$, and $\theta$ be the fractional parts of $(l(N)+m(N)) /(2 p),(l(N)-m(N)) /(2 p)$, $n(N) / p$, and $j / p$ respectively. Since $N /(2 \lambda+1) p \in E_{\lambda}\left(D^{(N)}\right)$, we have

$$
\left\{\frac{N}{(2 \lambda+1) p}\right\},\left\{\frac{\lambda N}{(2 \lambda+1) p}\right\} \in\left(\sqrt{2} \delta_{N}, 1-\sqrt{2} \delta_{N}\right) .
$$


Thus, any point $(\{x\},\{y\})$ satisfying

$$
\left|x-\frac{N}{(2 \lambda+1) p}\right|<\delta_{N} \text { and }\left|y-\frac{\lambda N}{(2 \lambda+1) p}\right|<\delta_{N}
$$

belongs to the set $D_{i}$ for some $1 \leq i \leq 3$. In particular, we have $(\eta, \omega)$, $\left(\eta^{\prime}, \omega\right) \in D_{i^{*}}$ for some $i^{*}$, since it is easily seen that

$$
\begin{gathered}
\left|\frac{l(N)-m(N)}{2 p}-\frac{N}{(2 \lambda+1) p}\right|<\frac{3}{2 p}<\frac{\delta_{N}}{2}, \\
\left|\frac{n(N)}{p}-\frac{N}{(2 \lambda+1) p}\right|<\frac{4}{3 p}<\frac{\delta_{N}}{3}
\end{gathered}
$$

and

$$
\left|\frac{l(N)+m(N)}{2 p}-\frac{\lambda N}{(2 \lambda+1) p}\right|<\frac{2}{3 p}<\frac{\delta_{N}}{6} .
$$

Now let $\nu(p) \geq 0$ be the largest integer such that $p^{\nu(p)}$ divides the integer (3.1). Then, since $p^{2}>2 N$, we obtain

$$
\begin{aligned}
\nu(p)= & {\left[\frac{l(N)+m(N)}{p}\right]-\left[\frac{j}{p}\right]-\left[\frac{l(N)+m(N)-j}{p}\right] } \\
& +\left[\frac{j+m(N)+n(N)}{p}\right]-\left[\frac{m(N)}{p}\right]-\left[\frac{j+n(N)}{p}\right] \\
& +\left[\frac{j+l(N)+n(N)}{p}\right]-\left[\frac{l(N)}{p}\right]-\left[\frac{j+n(N)}{p}\right] \\
= & {[2 \omega]-[2 \omega-\theta]+\left[\omega-\eta-\eta^{\prime}+\theta\right]-[\omega-\eta]-\left[\eta^{\prime}+\theta\right] } \\
& +\left[\omega+\eta+\eta^{\prime}+\theta\right]-[\omega+\eta]-\left[\eta^{\prime}+\theta\right] .
\end{aligned}
$$

We now distinguish three cases according to the value of $i^{*}$ as follows:

Case I $\left(i^{*}=1\right)$. Suppose that $\nu(p)=0$; then $[2 \omega]=[2 \omega-\theta]$ and $[\omega+\eta+$ $\left.\eta^{\prime}+\theta\right]=[\omega+\eta]+\left[\eta^{\prime}+\theta\right]$. Since $(\eta, \omega),\left(\eta^{\prime}, \omega\right) \in D_{1}$, we have $2 \omega>1$, $\omega+\eta<1, \omega+2 \eta>1$, and $\omega+2 \eta^{\prime}>1$; hence $\omega+\eta+\eta^{\prime}>1$. Thus $\eta^{\prime}+\theta \geq 1$ and $2 \omega \geq 1+\theta$; hence $2 \omega+\eta^{\prime} \geq 2$. This is a contradiction, since $\omega+\eta^{\prime}<1$. Therefore $\nu(p) \geq 1$.

Case II $\left(i^{*}=2\right)$. Suppose that $\nu(p)=0$; then $[2 \omega]=[2 \omega-\theta]$ and $[\omega-\eta+$ $\left.\eta^{\prime}+\theta\right]=[\omega-\eta]+\left[\eta^{\prime}+\theta\right]$. Since $(\eta, \omega),\left(\eta^{\prime}, \omega\right) \in D_{2}$, we have $2 \omega>1$, $\omega<\eta$, and $2 \omega+\eta^{\prime}<2$. On the other hand, it follows from (3.3) and (3.4) that

$$
\left|\eta-\eta^{\prime}\right| \leq\left|\eta-\left\{\frac{N}{(2 \lambda+1) p}\right\}\right|+\left|\eta^{\prime}-\left\{\frac{N}{(2 \lambda+1) p}\right\}\right|<\delta_{N} ;
$$

hence $\omega-\eta+\eta^{\prime}+\theta \geq 0$ for all sufficiently large $N$. Thus $2 \omega \geq 1+\theta$ and $\eta^{\prime}+\theta \geq 1$; hence $2 \omega+\eta^{\prime} \geq 2$, a contradiction. Therefore we have $\nu(p) \geq 1$.

Case III $\left(i^{*}=3\right)$. Suppose that $\nu(p)=0$; then $[2 \omega]=[2 \omega-\theta]$ and $[\omega-\eta+$ $\left.\eta^{\prime}+\theta\right]=[\omega-\eta]+\left[\eta^{\prime}+\theta\right]$. Since $(\eta, \omega),\left(\eta^{\prime}, \omega\right) \in D_{3}$, we have $\omega<\eta$ and $2 \omega+\eta^{\prime}<1$. On the other hand, it follows from (3.2) and (3.5) that

$$
\omega>\left\{\frac{\lambda N}{(2 \lambda+1) p}\right\}-\frac{\delta_{N}}{6}>\left(\sqrt{2}-\frac{1}{6}\right) \delta_{N}>\delta_{N} .
$$


Thus $\omega-\eta+\eta^{\prime}+\theta \geq 0$, since $\left|\eta-\eta^{\prime}\right|<\delta_{N}$. Hence $2 \omega \geq \theta$ and $\eta^{\prime}+\theta \geq 1$; so, $2 \omega+\eta^{\prime} \geq 1$, a contradiction. Therefore $\nu(p) \geq 1$.

Thus we have $\nu(p) \geq 1$ in any case. Since $j$ is arbitrary, the prime $p$ divides all the coefficients (3.1), as required.

We next study the asymptotic behavior of $\left\{d_{\lambda}(N)\right\}$ as $N \rightarrow \infty$. Let $L$ be a sufficiently large integer and let $I=(\alpha, \beta)$ be any connected component (open interval) of the open set $E_{\lambda}\left(D^{(L)}\right)$. Note that $I \subset E_{\lambda}\left(D^{(N)}\right)$ for any $N \geq L$. Then any prime number $p$ contained in the interval

$$
J=(N /(\beta(2 \lambda+1)), N /(\alpha(2 \lambda+1)))
$$

belongs to the set $H_{N}$ for any $N \geq L$. Therefore

$$
\sum_{\substack{p: \text { prime } \\ p \in J}} \log p \geq \pi\left(\frac{1}{\beta(2 \lambda+1)}, \frac{1}{\alpha(2 \lambda+1)} ; N\right) \log \left(\frac{N}{\beta(2 \lambda+1)}\right),
$$

where we denote by $\pi(a, b ; N)$ the number of prime numbers contained in the interval $(a N, b N)$. Using the well-known prime number theorem, we obtain

$$
\liminf _{N \rightarrow \infty} \frac{1}{N} \sum_{\substack{p: \text { prime } \\ p \in J}} \log p \geq \frac{1}{2 \lambda+1}\left(\frac{1}{\alpha}-\frac{1}{\beta}\right)=\frac{1}{2 \lambda+1} \int_{I} \frac{d x}{x^{2}} .
$$

Thus, for any positive number $c$, we have

$$
\liminf _{N \rightarrow \infty} \frac{1}{N} \log d_{\lambda}(N) \geq \frac{1}{2 \lambda+1} \int_{E_{c, \lambda, L}} \frac{d x}{x^{2}},
$$

where $E_{c, \lambda, L}=(0, c) \cap E_{\lambda}\left(D^{(L)}\right)$ is a finite union of open intervals. Hence we obtain the desired lower estimate by taking the limit as $c \rightarrow \infty$ and as $L \rightarrow \infty$. Similarly we can obtain the upper estimate using the inequality

$$
d_{\lambda}(N) \leq \prod_{p \in H_{N}^{*}} p
$$

where $H_{N}^{*}$ is the set of all prime numbers $p>\sqrt{2 N}$ satisfying $N /((2 \lambda+1) p) \epsilon$ $E_{\lambda}(D)$. This completes the proof.

For the proof of Theorem 1.1 we need the exact values of the integral

$$
e(\lambda)=\int_{E_{i}(D)} \frac{d x}{x^{2}}
$$

at integral points $\lambda=r \geq 2$. The following lemma enables us to calculate $e(r)$ exactly as a finite sum of the values of the digamma function $\psi(z)=\Gamma^{\prime}(z) / \Gamma(z)$ at rational points; hence $e(r)$ can be expressed as a finite sum of the values of elementary functions by Gauss' formula (Erdélyi et al. [6, p. 19]). A similar argument can be found in [7, p. 106]. For any $\lambda>1$ and any subset $W \subset$ $[0,1] \times[0,1]$, put $Y_{\lambda}(W)=E_{\lambda}(W) \cap(0,1)$.

Lemma 3.2. Let $r \geq 2$ be an integer. Then we have

$$
e(r)=\int_{Y_{r}(D)} d \psi(x)
$$


Proof. Since the function $\psi(x)$ is strictly increasing in $(0, \infty)$, the above Stieltjes integral certainly exists. Clearly $Y_{r}(D)$ is a finite union of disjoint open intervals. Since $\{r x\}=\{r\{x\}\}$ for any $x>0$, it follows that $x \in E_{r}(D)$ if and only if $\{x\} \in Y_{r}(D)$. This means that

$$
E_{r}(D)=\bigcup_{j=0}^{\infty}\left(j+Y_{r}(D)\right),
$$

where $j+Y_{r}(D)=\left\{j+x ; x \in Y_{r}(D)\right\}$. Let $(\alpha, \beta)$ be any connected component (open interval) of the set $Y_{r}(D)$. Since

$$
\sum_{j=0}^{\infty} \int_{j+\alpha}^{j+\beta} \frac{d x}{x^{2}}=\sum_{j=0}^{\infty}\left\{\frac{1}{j+\alpha}-\frac{1}{j+\beta}\right\}=\psi(\beta)-\psi(\alpha)=\int_{\alpha}^{\beta} d \psi(x),
$$

we obtain

$$
e(r)=\int_{E_{r}(D)} \frac{d x}{x^{2}}=\sum_{(\alpha, \beta) \in Y_{r}(D)} \int_{\alpha}^{\beta} d \psi(x)=\int_{Y_{r}(D)} d \psi(x) .
$$

This completes the proof.

Note that the set $Y_{r}(D)$ coincides with the projected set onto the $x$-axis of the intersection of $D$ and the line segments $y=\{r x\}$.

For example, we give the exact values of $e(3)$ and $e(4)$, which will be used in the proof of Theorem 1.1 in $\S 6$. It is easily seen that $Y_{3}(D)=\left(\frac{1}{5}, \frac{1}{4}\right) \cup\left(\frac{1}{3}, \frac{3}{7}\right) \cup$ $\left(\frac{2}{3}, \frac{5}{7}\right) \cup\left(\frac{5}{6}, \frac{6}{7}\right)$; hence, using Gauss' formula, we have

$$
\begin{aligned}
e(3)= & \frac{1}{4} \log \left[\left(\frac{1+\sqrt{5}}{2}\right)^{2 \sqrt{5}} 2^{-4} 3^{18} 5^{5} 7^{-14}\right] \\
& +\frac{\pi}{2}\left\{\cot \frac{\pi}{7}+\cot \frac{2 \pi}{7}-\cot \frac{3 \pi}{7}+\sqrt{1+\frac{2}{\sqrt{5}}}-1-\sqrt{3}\right\} .
\end{aligned}
$$

Similarly, since $Y_{4}(D)=\left(\frac{1}{6}, \frac{1}{5}\right) \cup\left(\frac{1}{4}, \frac{1}{3}\right) \cup\left(\frac{3}{8}, \frac{2}{5}\right) \cup\left(\frac{1}{2}, \frac{5}{9}\right) \cup\left(\frac{5}{8}, \frac{2}{3}\right) \cup\left(\frac{3}{4}, \frac{7}{9}\right) \cup\left(\frac{7}{8}, \frac{8}{9}\right)$, we have

$$
\begin{aligned}
e(4)= & \frac{1}{2} \log \left[(\sqrt{2}-1)^{2 \sqrt{2}} 2^{44} 3^{-18} 5^{-5}\right] \\
& +\frac{\pi}{2}\left\{\cot \frac{\pi}{9}+\cot \frac{2 \pi}{9}+\cot \frac{4 \pi}{9}+\sqrt{3}-1-\sqrt{2}-\sqrt{2+\frac{2}{\sqrt{5}}}\right\} .
\end{aligned}
$$

Numerically one has $e(3)=2.0161878 \ldots$ and $e(4)=2.8124484 \ldots$.

Moreover, in order to obtain better irrationality measures of $L_{2}(1 / k)$, we also need the exact values of $e(\lambda)$ at rational points $\lambda=r / s$.

Lemma 3.3. Let $r>s$ be positive integers with $(r, s)=1$. Let $T_{j}$ be the affine mapping in $\mathbf{R}^{2}$ defined by $T_{j}:(x, y) \rightarrow((x+j) / s, y)$ for $0 \leq j \leq s-1$ and put $D[s]=T_{0}(D) \cup T_{1}(D) \cup \cdots \cup T_{s-1}(D)$. Then we have

$$
e\left(\frac{r}{s}\right)=\frac{1}{s} \int_{Y_{r}(D[s])} d \psi(x) .
$$


Proof. Substituting $x=s y$, we have

$$
e\left(\frac{r}{s}\right)=\int_{E_{r / s}(D)} \frac{d x}{x^{2}}=\frac{1}{s} \int_{E^{*}} \frac{d y}{y^{2}},
$$

where $E^{*}=\{x>0 ;(\{s x\},\{r x\}) \in D\}$. Since $s\{x\}-\{s x\}$ is a nonnegative integer less than $s$, it follows that $(\{s x\},\{r x\}) \in D$ if and only if $(\{x\},\{r x\}) \in D[s]$; hence $E^{*}=E_{r}(D[s])$. Using the same method as in the previous proof, we thus obtain

$$
\int_{E^{*}} \frac{d y}{y^{2}}=\int_{E_{r}(D[s])} \frac{d y}{y^{2}}=\int_{Y_{r}(D[s])} d \psi(x),
$$

as required.

The Stieltjes integral in the above lemma takes a fairly complicated form when we express it as a finite sum of the values of the digamma function at rational points. We put $\lambda=r / s, \mathbf{a}_{u}=\left(b_{u}, c_{u}\right)$, and

$$
A_{u}=\left\{(i, j) \in \mathbf{Z} \times \mathbf{Z} ; 0 \leq i \leq s-1,\left[i \lambda+b_{u}\right] \leq j \leq\left[i \lambda+c_{u}\right]\right\}
$$

for $1 \leq u \leq 6$, where

$$
\begin{gathered}
\mathbf{a}_{1}=\left(1, \frac{\lambda-1}{3}\right), \quad \mathbf{a}_{2}=\left(\frac{\lambda+2}{3}, \lambda\right), \quad \mathbf{a}_{3}=\left(0, \frac{\lambda-2}{4}\right), \\
\mathbf{a}_{4}=\left(\frac{\lambda+1}{4}, \frac{\lambda-1}{2}\right), \quad \mathbf{a}_{5}=\left(\frac{\lambda+1}{2}, \frac{2 \lambda-2}{3}\right), \quad \mathbf{a}_{6}=\left(\frac{2 \lambda+1}{3}, \frac{2 \lambda-1}{3}\right) .
\end{gathered}
$$

Then we obtain

$$
\begin{aligned}
& e\left(\frac{r}{s}\right)=\frac{1}{s}\left[\sum_{1} \psi\left(\frac{j-i}{r-s}\right)+\sum_{2} \psi\left(\frac{1+i+j}{r+s}\right)\right. \\
&+\sum_{3} \psi\left(\frac{1+i+2 j}{2 r+s}\right)+\sum_{4} \psi\left(\frac{2+i+2 j}{2 r+s}\right) \\
&\left.-\sum_{5} \psi\left(\frac{j}{r}\right)-\sum_{6} \psi\left(\frac{1+2 j}{2 r}\right)-\sum_{7} \psi\left(\frac{1+2 i+j}{r+2 s}\right)\right],
\end{aligned}
$$

where $\sum_{v}$ denotes the summation over $(i, j) \in B_{v} \quad(1 \leq v \leq 7)$ with $B_{1}=$ $A_{1} \cup A_{5}, B_{2}=A_{3} \cup A_{5}, B_{3}=A_{2}, B_{4}=A_{6}, B_{5}=A_{1} \cup A_{2}, B_{6}=A_{4} \cup A_{5} \cup A_{6}$, and $B_{7}=A_{3}$.

Note that $e(\lambda)$ is a continuous function of $\lambda$, since the closure of $E_{\lambda}(D)$ is continuous with respect to $\lambda$ in the sense of the Hausdorff metric.

\section{PADÉ-TYPe APPROXIMATIONS}

We now construct explicitly our rational approximations to the dilogarithm $L_{2}(z)$ using the polynomials (2.2).

Let $M_{N}$ be the least common multiple of $\{1,2, \ldots, N\}$. For any $z \in$ $\mathrm{C}-[1, \infty)$, we consider the following double integral:

$$
Q_{\lambda, N}\left(\frac{1}{z}\right)=\int_{0}^{1} \int_{0}^{1} \frac{P_{\lambda, N}\left(\frac{1}{z}\right)-P_{\lambda, N}(x y)}{\frac{1}{z}-x y} d x d y .
$$


Clearly $Q_{\lambda, N}(w)$ is a polynomial (of $w$ ) of degree less than $N$ with rational coefficients such that $M_{N}^{2} Q_{\lambda, N}(w) \in \mathbf{Z}[w]$. Then, from (4.1),

$$
Q_{\lambda, N}\left(\frac{1}{z}\right)=P_{\lambda, N}\left(\frac{1}{z}\right) L_{2}(z)-J_{\lambda, N}\left(\frac{1}{z}\right),
$$

where

$$
J_{\lambda, N}(w)=\int_{0}^{1} \int_{0}^{1} \frac{P_{\lambda, N}(x y)}{w-x y} d x d y=-\int_{0}^{1} \frac{P_{\lambda, N}(t)}{w-t} \log t d t .
$$

Thus, putting $P_{\lambda, N}^{*}(z)=z^{N} P_{\lambda, N}(1 / z)$ and $Q_{\lambda, N}^{*}(z)=z^{N} Q_{\lambda, N}(1 / z)$, we obtain

$$
\begin{aligned}
P_{\lambda, N}^{*}(z) L_{2}(z)-Q_{\lambda, N}^{*}(z) & \\
= & z^{N} J_{\lambda, N}\left(\frac{1}{z}\right)=-z^{N+1} \int_{0}^{1} \frac{P_{\lambda, N}(t)}{1-z t} \log t d t \\
= & -\sum_{j=m(N)}^{\infty} z^{j+N+1} \int_{0}^{1} t^{j} P_{\lambda, N}(t) \log t d t \\
= & O\left(z^{m(N)+N+1}\right)=O\left(z^{[3 \lambda N /(2 \lambda+1)]}\right),
\end{aligned}
$$

where $P_{\lambda, N}^{*}(z)$ and $Q_{\lambda, N}^{*}(z)$ are polynomials of degrees less than or equal to $N-n(N)$ and $N$ respectively with rational coefficients such that $P_{\lambda, N}^{*}(z) \in \mathbf{Z}[z]$ and $M_{N}^{2} Q_{\lambda, N}^{*}(z) \in \mathbf{Z}[z]$.

Equality (4.3) provides a one-parameter family $(1<\lambda<\infty)$ of Padé-type approximations to the dilogarithm. The extreme case $\lambda=\infty$ was completely investigated by the author [8]; indeed, $P_{\infty, N}(x)$ coincides with the polynomial $A_{N}(x)$ defined in (1.1), which gives the best order $O\left(z^{[3 N / 2]}\right)$ in the right-hand side of (4.3). Nevertheless, owing to the arithmetical property stated in Lemma $3.1,(4.3)$ can provide better approximations for a suitable choice of $\lambda$ 's.

Similarly our polynomials $P_{\lambda, N}(x)$ also provide rational approximations to the logarithm $\log (1-z)$; indeed, using the integral

$$
R_{\lambda, N}\left(\frac{1}{z}\right)=-\int_{0}^{1} \frac{P_{\lambda, N}\left(\frac{1}{z}\right)-P_{\lambda, N}(t)}{\frac{1}{z}-t} d t
$$

for any $z \in \mathbf{C}-[1, \infty)$, we obtain

$$
R_{\lambda, N}\left(\frac{1}{z}\right)=P_{\lambda, N}\left(\frac{1}{z}\right) \log (1-z)-I_{\lambda, N}\left(\frac{1}{z}\right),
$$

where

$$
I_{\lambda, N}(w)=\int_{0}^{1} \frac{P_{\lambda, N}(t)}{t-w} d t
$$

Thus, putting $R_{\lambda, N}^{*}(z)=z^{N} R_{\lambda, N}(1 / z)$, we have

$$
\begin{aligned}
P_{\lambda, N}^{*}(z) & \log (1-z)-R_{\lambda, N}^{*}(z) \\
= & z^{N} I_{\lambda, N}\left(\frac{1}{z}\right)=-z^{N+1} \int_{0}^{1} \frac{P_{\lambda, N}(t)}{1-z t} d t \\
= & -\sum_{j=l(N)}^{\infty} z^{j+N+1} \int_{0}^{1} t^{j} P_{\lambda, N}(t) d t \\
= & O\left(z^{l(N)+N+1}\right)=O\left(z^{[(3 \lambda+2) N /(2 \lambda+1)]}\right),
\end{aligned}
$$


where $R_{\lambda, N}^{*}(z)$ is a polynomial of degree less than or equal to $N$ with rational coefficients such that $M_{N} R_{\lambda, N}^{*}(z) \in \mathbf{Z}[z]$. (4.3) and (4.5) will be used to show the linear independence of the numbers $1, \log (1-1 / k)$, and $L_{2}(1 / k)$.

\section{Estimates of $P_{\lambda, N}(k)$ AND $J_{\lambda, N}(k)$}

Let $k \neq 0,1$ be an arbitrarily fixed integer. The aim of this section is to give good upper estimates of $\left|P_{\lambda, N}(k)\right|$ and $\left|J_{\lambda, N}(k)\right|$ for all sufficiently large $N$. Throughout this section we write $l=l(N), m=m(N)$, and $n=n(N)$ for brevity.

We first estimate $P_{\lambda, N}(k)$. For any real number $\lambda>1$, the algebraic equation

$$
x((2 \lambda+1) x-1)^{2}-k \lambda(3 x-1)((3 \lambda+2) x-\lambda-2)=0
$$

has a unique real root $\alpha_{\lambda, k}$ in the interval $(k, \infty)$ or $(-\infty, k)$ according as $k \geq 2$ or $k \leq-1$ respectively. For any $x \in \mathbf{R}$, we also define

$$
\begin{aligned}
F_{\lambda}(x)= & \log |x|+(\lambda-1) \log \left|\frac{\lambda(3 x-1)}{\lambda-1}\right| \\
& +(\lambda+1) \log \left|\frac{(3 \lambda+2) x-\lambda-2}{\lambda+1}\right| .
\end{aligned}
$$

Then our result concerning $P_{\lambda, N}(k)$ can be stated as follows:

Lemma 5.1. For any integer $k \neq 0,1$, we have

$$
\limsup _{N \rightarrow \infty} \frac{1}{N} \log \left|P_{\lambda, N}(k)\right| \leq \frac{1}{2 \lambda+1} F_{\lambda}\left(\alpha_{\lambda, k}\right) .
$$

Proof. By Cauchy's integral formula,

$$
P_{\lambda, N}(k)=\left(\frac{1}{2 \pi i}\right)^{2} \int_{C} \frac{z^{l}}{(z-k)^{l+1}} \int_{C^{\prime}} \frac{w^{m+n}(1-w)^{l+m}}{(w-z)^{m+1}} d w d z,
$$

where $C$ and $C^{\prime}$ are the circles centered at $z=k$ and $w=z$ with radii $\rho$ and $\xi$ respectively. ( $\rho$ and $\xi$ are positive parameters to be determined later.) Then it follows immediately that

$$
\limsup _{N \rightarrow \infty} \frac{1}{N} \log \left|P_{\lambda, N}(k)\right| \leq \frac{1}{2 \lambda+1} S_{\lambda, k}(\rho, \xi),
$$

where

$$
\begin{aligned}
S_{\lambda, k}(\rho, \xi)= & (\lambda+1) \log \left(1+\frac{|k|}{\rho}\right)-(\lambda-1) \log \xi \\
& +\lambda \log (|k|+\rho+\xi)+2 \lambda \log (|k-1|+\rho+\xi) .
\end{aligned}
$$

Now it is easily verified that the equations

$$
\frac{\partial}{\partial \rho} S_{\lambda, k}(\rho, \xi)=\frac{\partial}{\partial \xi} S_{\lambda, k}(\rho, \xi)=0
$$

have a unique solution $\left(\rho_{0}, \xi_{0}\right)$ in the region $(0, k) \times(0,2 k)$ satisfying

$$
\xi_{0}=\frac{(\lambda-1) \rho_{0}\left(\rho_{0}+|k|\right)}{(\lambda+1)|k|} .
$$


Moreover, the point

$$
t_{0}=\frac{(\lambda+1)|k|-\rho_{0}}{(\lambda+1)|k|-(2 \lambda+1) \rho_{0}}
$$

satisfies the algebraic equation (5.1); therefore $t_{0}=\alpha_{\lambda, k}$ since $t_{0}=k+$ $\operatorname{sgn}(k)\left(\rho_{0}+\xi_{0}\right)$. Thus it can be seen that $S_{\lambda, k}\left(\rho_{0}, \xi_{0}\right)=F_{\lambda}\left(\alpha_{\lambda, k}\right)$. This completes the proof.

We next estimate $J_{\lambda, N}(k)$. This estimate is the most essential part in this paper and we must distinguish two cases according to the sign of the value of $k$. We first consider the case $k \geq 2$. In this case, the algebraic equation (5.1) has two real roots $\beta_{\lambda, k}$ and $\gamma_{\lambda, k}$ in the interval $\left(0, \frac{1}{3}\right)$ and $((\lambda+2) /(3 \lambda+2), 1)$ respectively. Then we have

Lemma 5.2. For any integer $k \geq 2$, we have

$$
\limsup _{N \rightarrow \infty} \frac{1}{N} \log \left|J_{\lambda, N}(k)\right| \leq \frac{1}{2 \lambda+1} \max \left\{F_{\lambda}\left(\beta_{\lambda, k}\right), F_{\lambda}\left(\gamma_{\lambda, k}\right)\right\} .
$$

Proof. After an $l$-fold partial integration, we have

$$
\begin{aligned}
J_{\lambda, N}(k) & =-\int_{0}^{1} \frac{P_{\lambda, N}(x)}{k-x} \log x d x \\
& =\frac{(-1)^{l+1}}{l ! m !} \int_{0}^{1} x^{l}\left(x^{m+n}(1-x)^{l+m}\right)^{(m)}\left(\frac{\log x}{k-x}\right)^{(l)} d x .
\end{aligned}
$$

On the other hand, for an arbitrarily fixed $x \in(0,1]$,

$$
\begin{aligned}
\frac{1}{l !}\left(\frac{\log x}{k-x}\right)^{(l)} & =\frac{\log k}{l !}\left(\frac{1}{k-x}\right)^{(l)}+\frac{1}{l !}\left(\frac{\log (x / k)}{k-x}\right)^{(l)} \\
& =\frac{\log k}{(k-x)^{l+1}}+\frac{1}{2 \pi i} \int_{C} \frac{1}{(z-x)^{l+1}} \cdot \frac{\log (z / k)}{k-z} d z
\end{aligned}
$$

where $C$ is a circle centered at $z=x$ with radius less than 1 . Since the point $z=k$ is a removable singularity of the function $f(z)=\log (z / k) /(k-z)$, we can change the contour $C$ to the new one $C_{R}$ consisting of the circle centered at the origin with radius $R>1$ and two segments joining $z=-R$ and the origin (see Figure 3 on the next page). Letting $R \rightarrow \infty$ and substituting $y=x /(t+x)$ for an arbitrarily fixed $x \in(0,1]$, we obtain

$$
\begin{aligned}
\frac{1}{2 \pi i} \int_{C} \frac{f(z)}{(z-x)^{l+1}} d z & =(-1)^{l} \int_{0}^{\infty} \frac{d t}{(t+x)^{l+1}(k+t)} \\
& =\left(-\frac{1}{x}\right)^{l} \int_{0}^{1} \frac{y^{l}}{k y+x(1-y)} d y .
\end{aligned}
$$

Hence, combining (5.2), (5.3), and (5.4),

$$
\begin{aligned}
J_{\lambda, N}(k)= & \frac{(-1)^{l+1} \log k}{m !} \int_{0}^{1} \frac{x^{l}}{(k-x)^{l+1}}\left(x^{m+n}(1-x)^{l+m}\right)^{(m)} d x \\
& -\frac{1}{m !} \int_{0}^{1} \int_{0}^{1} \frac{y^{l}}{k y+x(1-y)}\left(x^{m+n}(1-x)^{l+m}\right)^{(m)} d x d y \\
\equiv & (-1)^{l+1}(\log k) \Lambda_{N}(k)-\Omega_{N}(k), \quad \text { say . }
\end{aligned}
$$




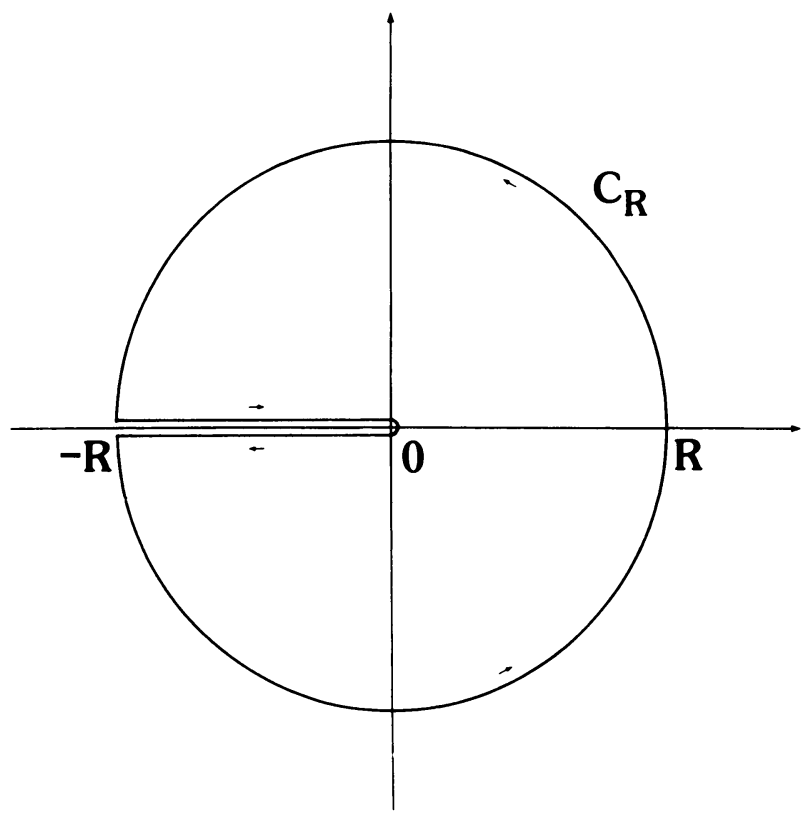

FIGURE 3. The contour $C_{R}$

We first estimate the integral $\Lambda_{N}(k)$. After an $m$-fold partial integration, we have

$$
\Lambda_{N}(k)=\frac{(-1)^{m}}{m !} \int_{0}^{1} x^{m+n}(1-x)^{l+m}\left(\frac{x^{l}}{(k-x)^{l+1}}\right)^{(m)} d x .
$$

By Cauchy's integral formula, we find

$$
\frac{1}{m !}\left(\frac{x^{l}}{(k-x)^{l+1}}\right)^{(m)}=\frac{1}{2 \pi i} \int_{C^{\prime}} \frac{z^{l}}{(z-x)^{m+1}(k-z)^{l+1}} d z,
$$

where $C^{\prime}$ is a small circle centered at $z=x$. Substituting $w=z /(z-k)$, we thus obtain

$$
\Lambda_{N}(k)=\frac{(-1)^{m+1}}{2 \pi i} \int_{0}^{1} x^{m+n}(1-x)^{l+m} \int_{C^{\prime \prime}} G_{k, N}(x, w) d w d x,
$$

where

$$
G_{k, N}(x, w)=\frac{w^{l}(1-w)^{m}}{(k w+x(1-w))^{m+1}}
$$

and $C^{\prime \prime}$ is the circle centered at $w=x /(x-k)$ with radius $\rho x /(k-\rho x)-$ $x /(k-x) .(\rho \in(1, k / x)$ is a real parameter to be determined later.) Since the maximum of $\left|G_{k, N}(x, w)\right|$ is attained at $w=\rho x /(\rho x-k)$, it follows from (5.5) that

$$
\limsup _{N \rightarrow \infty} \frac{1}{N} \log \left|\Lambda_{N}(k)\right| \leq \frac{1}{2 \lambda+1} \max _{0<x<1} T_{\lambda, k}(x, \rho),
$$

where

$$
\begin{aligned}
T_{\lambda, k}(x, \rho)= & (\lambda+2) \log x+2 \lambda \log (1-x) \\
& -(\lambda-1) \log (\rho-1)+(\lambda+1) \log \left(\frac{\rho}{k-\rho x}\right) .
\end{aligned}
$$


Then it is easily verified that $\frac{\partial}{\partial x} T_{\lambda, k}(x, \rho)=0$ has a unique root $x \in(0,1)$ for each fixed $\rho \in(1, k)$ and that the equations

$$
\frac{\partial}{\partial x} T_{\lambda, k}(x, \rho)=\frac{\partial}{\partial \rho} T_{\lambda, k}(x, \rho)=0
$$

have a unique solution $\left(x_{0}, \rho_{0}\right)$ in the region $\left(\frac{1}{3}, 1\right) \times(1, k)$ satisfying

$$
\rho_{0}=\frac{(2 \lambda+1) x_{0}-1}{\lambda\left(3 x_{0}-1\right)} \text {. }
$$

Moreover, the point $x_{0}$ satisfies the algebraic equation (5.1); hence $x_{0}=\gamma_{\lambda, k}$ since $\frac{1}{3}<x_{0}<1$. We thus obtain $T_{\lambda, k}\left(x_{0}, \rho_{0}\right)=F_{\lambda}\left(\gamma_{\lambda, k}\right)$.

We next estimate the integral $\Omega_{N}(k)$. After an $m$-fold partial integration with respect to $x$, we have

$$
\Omega_{N}(k)=(-1)^{m} \int_{0}^{1} \int_{0}^{1} x^{m+n}(1-x)^{l+m} G_{k, N}(x, y) d x d y
$$

hence

$$
\limsup _{N \rightarrow \infty} \frac{1}{N} \log \left|\Omega_{N}(k)\right|=\frac{1}{2 \lambda+1} \max _{\substack{0<x<1 \\ 0<y<1}} U_{\lambda, k}(x, y),
$$

where

$$
\begin{aligned}
U_{\lambda, k}(x, y)= & \lambda \log x+2 \lambda \log (1-x)+(\lambda+1) \log y \\
& +(\lambda-1) \log \left(\frac{1-y}{k y+x(1-y)}\right) .
\end{aligned}
$$

Then it is easily seen that the equations

$$
\frac{\partial}{\partial x} U_{\lambda, k}(x, y)=\frac{\partial}{\partial y} U_{\lambda, k}(x, y)=0
$$

have a unique solution $\left(x_{0}, y_{0}\right)$ in the region $\left(0, \frac{1}{3}\right) \times(0,1)$ satisfying

$$
y_{0}=\frac{\lambda+2-(3 \lambda+2) x_{0}}{(\lambda+1)\left(1-x_{0}\right)} \text {. }
$$

Moreover, the point $x_{0}$ satisfies the algebraic equation (5.1); hence $x_{0}=\beta_{\lambda, k}$ since $0<x_{0}<\frac{1}{3}$. We thus obtain $U_{\lambda, k}\left(x_{0}, y_{0}\right)=F_{\lambda}\left(\beta_{\lambda, k}\right)$. Therefore, from (5.6) and (5.8),

$$
\limsup _{N \rightarrow \infty} \frac{1}{N} \log \left|J_{\lambda, N}(k)\right| \leq \frac{1}{2 \lambda+1} \max \left\{F_{\lambda}\left(\beta_{\lambda, k}\right), F_{\lambda}\left(\gamma_{\lambda, k}\right)\right\} .
$$

This completes the proof.

We next consider the case $k \leq-1$. In this case we will find ourselves in a different situation from the previous case. So we restrict ourselves to the following two cases:

$$
\begin{array}{ll}
\text { (a) } \kappa \geq \frac{\lambda^{2}+\lambda-1}{3 \lambda^{2}+\lambda-1} ; & \text { (b) } \kappa \leq \frac{2 \lambda^{2}+2 \lambda-1}{6 \lambda^{2}+4 \lambda-1},
\end{array}
$$

where we put $\kappa \equiv \kappa(\lambda, k)=|k| /\left(\lambda^{2}-1\right)$ for brevity. In case (a) it will be shown that the algebraic equation (5.1) has two positive roots $\beta_{\lambda, k}<\gamma_{\lambda, k}$ in 
the interval $\left(\frac{1}{3},(\lambda+2) /(3 \lambda+2)\right)$. On the other hand, in case $(b)$, the equation (5.1) has no real roots except for $\alpha_{\lambda, k}$. So we introduce the following quadratic equation instead of $(5.1)$ :

$$
x((2 \lambda+1) x-1)-k((3 \lambda+1) x-\lambda-1)=0,
$$

which has a unique positive root $\delta_{\lambda, k}$ in the interval $(\kappa, 1)$. For any $x \in$ $(0,1)$, we also put

$$
H_{\lambda}(x)=\log x+\lambda \log \left(\frac{1-x}{\lambda}\right)+\lambda \log |(3 \lambda+1) x-\lambda-1| .
$$

Lemma 5.3. Let $k$ be a negative integer. Then

(1) for any $\lambda>1$ satisfying condition (a), we have

$$
\limsup _{N \rightarrow \infty} \frac{1}{N} \log \left|J_{\lambda, N}(k)\right| \leq \frac{1}{2 \lambda+1} F_{\lambda}\left(\beta_{\lambda, k}\right)
$$

(2) for any $\lambda>1$ satisfying condition (b), we have

$$
\limsup _{N \rightarrow \infty} \frac{1}{N} \log \left|J_{\lambda, N}(k)\right| \leq \frac{1}{2 \lambda+1} H_{\lambda}\left(\delta_{\lambda, k}\right) .
$$

Proof. In the previous proof we had shown that

$$
J_{\lambda, N}(\zeta)=(-1)^{l+1}(\log \zeta) \Lambda_{N}(\zeta)-\Omega_{N}(\zeta)
$$

for every integer $\zeta$ greater than 1 . However it is easily seen that (5.11) still holds for every real $\zeta>1$. Moreover, for any $\zeta>2$, we can change the contour $C^{\prime \prime}$ with respect to $w$ in (5.5) to the unit circle $C_{0}$ centered at the origin and the contour $[0,1]$ with respect to $y$ in $(5.7)$ to the upper semicircle $\Gamma_{0}$ centered at $y=\frac{1}{2}$ with radius $\frac{1}{2}$, since $-1<x /(x-\zeta)<0$ for any $(x, \zeta) \in(0,1] \times(2, \infty)$. Thus we have

$$
\Lambda_{N}(\zeta)=\frac{(-1)^{m+1}}{2 \pi i} \int_{0}^{1} x^{m+n}(1-x)^{l+m} \int_{C_{0}} G_{\zeta, N}(x, w) d w d x
$$

and

$$
\Omega_{N}(\zeta)=(-1)^{m} \int_{0}^{1} x^{m+n}(1-x)^{l+m} \int_{\Gamma_{0}} G_{\zeta, N}(x, w) d w d x .
$$

Then it is easily verified that $\Lambda_{N}(\zeta)$ is analytic in the region $\dot{\mathbf{C}}_{0}=\{\zeta ;|\zeta-1|>$ $1\}$ and that $\Omega_{N}(\zeta)$ is analytic in the region $\mathbf{C}_{1}=\mathbf{C}-\{\zeta=t i ; t \geq 0\}$. Since $J_{\lambda, N}(\zeta)$ is clearly analytic in $\mathbf{C}_{2}=\mathbf{C}-[0,1]$ and since the region $\mathbf{C}_{0} \cap \mathbf{C}_{1} \cap \mathbf{C}_{2}$ contains the half-line $\{\zeta=t ; t>2\}$, it follows that (5.11) holds for every $\zeta \in \mathbf{C}_{0} \cap \mathbf{C}_{1} \cap \mathbf{C}_{2}$. (Here, of course, we regard $\log \zeta$ as an analytic function in $\mathrm{C}_{1}$; that is, $-3 \pi / 2<\operatorname{Im}(\log \zeta)=\arg \zeta<\pi / 2$.) In particular, putting $\zeta=k$ for any negative integer $k$, we have

$$
J_{\lambda, N}(k)=(-1)^{l+1}(\log |k|-\pi i) \Lambda_{N}(k)-\Omega_{N}(k) .
$$

For brevity, put $\nu \equiv \nu(k, x)=x /(x+|k|)$. Clearly $\nu \in(0,1)$ is the pole of the rational function $G_{k, N}(x, w)$ for an arbitrarily fixed $x \in(0,1]$. Hence, from (5.12), (5.13), and (5.14),

$$
\limsup _{N \rightarrow \infty} \frac{1}{N} \log \left|J_{\lambda, N}(k)\right| \leq \frac{1}{2 \lambda+1} \log \left[\max _{0 \leq x \leq 1} \frac{x^{\lambda}(1-x)^{2 \lambda}}{(x+|k|)^{\lambda-1}} f_{\lambda, k}(x)\right],
$$


where

$$
f_{\lambda, k}(x)=\min _{C, \Gamma} \max _{z \in C \cup \Gamma} g_{\lambda, \nu}(z), \quad g_{\lambda, \nu}(z)=|z|^{\lambda+1}\left|\frac{z-1}{z-\nu}\right|^{\lambda-1},
$$

and the minimum extends over all simple closed curves $C$ enclosing the point $\nu$ and all simple arcs $\Gamma$ joining $z=0$ and $z=1$, homotopic to $\Gamma_{0}$ in the punctured region $\mathbf{C}-\{\nu\}$. Since $g_{\lambda, \nu}(z)=g_{\lambda, \nu}(\bar{z})$ and $\Gamma \cup \bar{\Gamma}$ is a closed curve enclosing the point $\nu, f_{\lambda, k}(x)$ can be expressed simply as

$$
f_{\lambda, k}(x)=\min _{\Gamma} \max _{z \in \Gamma} g_{\lambda, \nu}(z) \text {. }
$$

Put $\kappa^{*}=\min (\kappa, 1)$ for brevity. We first suppose that $0<x \leq \kappa^{*}$. Then it is easily verified that $g_{\lambda, \nu}(z)$ has two real saddle points

$$
z_{ \pm} \equiv z_{ \pm}(\lambda, \nu)=\frac{1}{\lambda+1}\left(\lambda \nu+1 \pm \sqrt{(1-\nu)\left(1-\lambda^{2} \nu\right)}\right)
$$

respectively, satisfying $\nu<z_{-} \leq \sqrt{\nu} \leq z_{+}<1$. Moreover, one can certainly find some simple arc $\Gamma^{*}$ such that the maximum of $g_{\lambda, \nu}(z)$, as $z$ varies in $\Gamma^{*}$, is attained at the larger saddle point $z_{+}$. Hence

$$
f_{\lambda, k}(x) \leq g_{\lambda, \nu}\left(z_{+}\right)=\max _{\sqrt{\nu} \leq y<1} g_{\lambda, \nu}(y) .
$$

We next suppose that $\kappa<1$ and $\kappa<x<1$. Then it is easily seen that $g_{\lambda, \nu}(z)$ has two complex saddle points

$$
\zeta_{ \pm} \equiv \zeta_{ \pm}(\lambda, \nu)=\frac{1}{\lambda+1}\left(\lambda \nu+1 \pm i \sqrt{(1-\nu)\left(\lambda^{2} \nu-1\right)}\right)
$$

respectively, satisfying $\left|\zeta_{ \pm}\right|=\sqrt{\nu}$. Moreover, one can easily find a suitable simple arc $\Gamma_{*}$ such that the maximum of $g_{\lambda, \nu}(z)$, as $z$ varies in $\Gamma_{*}$, is attained at $z=\zeta_{+}$. Hence

$$
f_{\lambda, k}(x) \leq g_{\lambda, \nu}\left(\zeta_{+}\right)=\nu=g_{\lambda, \nu}(\sqrt{\nu})=\max _{\sqrt{\nu} \leq y<1} g_{\lambda, \nu}(y)
$$

Therefore we have, from (5.15) and (5.16),

$$
\limsup _{N \rightarrow \infty} \frac{1}{N} \log \left|J_{\lambda, N}(k)\right| \leq \frac{1}{2 \lambda+1} \max _{0<x<1} \max _{\sqrt{\nu} \leq y<1} V_{\lambda, k}(x, y),
$$

where

$$
\begin{aligned}
V_{\lambda, k}(x, y)= & \lambda \log x+2 \lambda \log (1-x)+(\lambda+1) \log y \\
& +(\lambda-1) \log \left(\frac{1-y}{(x+|k|) y-x}\right) .
\end{aligned}
$$

Now it is easily verified that the curve defined by $\frac{\partial}{\partial y} V_{\lambda, k}(x, y)=0$ in the region $D^{*}=\{(x, y) ; 0<x<1, \nu \leq y<1\}$ can be expressed by the following two continuous functions:

$$
h_{ \pm}(x)=z_{ \pm}\left(\lambda, \frac{x}{x+|k|}\right) \text { for } 0<x \leq \kappa^{*}
$$

respectively. More precisely, $h_{-}(x)<y<h_{+}(x)$ if and only if $\frac{\partial}{\partial y} V_{\lambda, k}(x, y)>$ 0 . The functions $h_{ \pm}(x)$, which are also defined for $0<x \leq \kappa$, satisfy $\nu<$ 
$h_{-}(x) \leq \sqrt{\nu} \leq h_{+}(x)$ and $h_{+}(\kappa)=h_{-}(\kappa)=1 / \lambda .\left(h_{+}(x)\right.$ is strictly decreasing and $h_{-}(x)$ is strictly increasing for $0<x \leq \kappa$.) On the other hand, the curve defined by $\frac{\partial}{\partial x} V_{\lambda, k}(x, y)=0$ in the region $D^{*}$ can be expressed by the following rational function:

$$
\eta(x)=\frac{x((2 \lambda+1) x-1)}{(2 \lambda+1) x^{2}+(3 \lambda|k|-1) x-\lambda|k|} \quad \text { for } \frac{1}{3}<x<1 .
$$

Clearly $y<\eta(x)$ if and only if $\frac{\partial}{\partial x} V_{\lambda, k}(x, y)>0$. The minimum of $\eta(x)$ for $x \geq \frac{1}{3}$ is attained at

$$
\theta(\lambda)=\frac{1}{3}\left(1+\sqrt{\frac{2 \lambda-2}{2 \lambda+1}}\right) \in\left(\frac{1}{3}, \frac{2}{3}\right) .
$$

$\left(\eta(x)\right.$ is strictly decreasing for $\frac{1}{3}<x \leq \theta(\lambda)$ and strictly increasing for $x \geq$ $\theta(\lambda)$.)

We first consider the case in which $\eta(\kappa) \leq h_{ \pm}(\kappa)=1 / \lambda$. Then it is easily seen that this inequality is equivalent to condition (a). In this case, the equations $h_{ \pm}(x)=\eta(x)$ have obviously at least one solution in $\left(\frac{1}{3}, \kappa\right)$ respectively, since $h_{+}(0)>1 / \lambda, h_{-}(0)=0$, and $\eta\left(\frac{1}{3}\right)=1$. On the other hand, it can be seen that any solution $x>\frac{1}{3}$ of the equations $h_{ \pm}(x)=\eta(x)$ must satisfy the algebraic equation (5.1). Since (5.1) has at most two positive roots, it follows immediately that $(5.1)$ has exactly two distinct positive roots $\beta_{\lambda, k}<\gamma_{\lambda, k}$ such that $h_{+}\left(\beta_{\lambda, k}\right)=\eta\left(\beta_{\lambda, k}\right)$ and $h_{-}\left(\gamma_{\lambda, k}\right)=\eta\left(\gamma_{\lambda, k}\right)$ with $\frac{1}{3}<\beta_{\lambda, k}<\gamma_{\lambda, k}<(\lambda+2) /(3 \lambda+2)$. Hence the maximum of $V_{\lambda, k}(x, y)$ in the region $D_{*}=\left\{(x, y) ; 0<x \leq \kappa^{*}, \sqrt{\nu} \leq y<1\right\}$ is attained at the point $\left(\beta_{\lambda, k}, \eta\left(\beta_{\lambda, k}\right)\right)$, which is a unique solution of the equations

$$
\frac{\partial}{\partial x} V_{\lambda, k}(x, y)=\frac{\partial}{\partial y} V_{\lambda, k}(x, y)=0
$$

in $D_{*}$; we thus have

$$
\max _{0<x \leq \kappa^{*}} \max _{\sqrt{\nu} \leq y<1} V_{\lambda, k}(x, y)=F_{\lambda}\left(\beta_{\lambda, k}\right) .
$$

Statement (1) of the lemma is thus proved if $\kappa \geq 1$. On the other hand, if $\kappa<1$, then we must consider the function

$$
\begin{aligned}
W_{\lambda, k}(x) & =V_{\lambda, k}\left(x, \sqrt{\frac{x}{x+|k|}}\right) \\
& =(\lambda+1) \log x+2 \lambda \log (1-x)-\lambda \log (x+|k|)
\end{aligned}
$$

for $\kappa \leq x<1$. Clearly the equation $W_{\lambda, k}^{\prime}(x)=0$ is equivalent to the quadratic equation (5.9) for $0<x<1$; thus $W_{\lambda, k}(x)$ is strictly increasing for $0<x<$ $\delta_{\lambda, k}$ and strictly decreasing for $\delta_{\lambda, k}<x<1$. Moreover,

$$
\begin{aligned}
W_{\lambda, k}^{\prime}(\kappa) & =\frac{\partial}{\partial x} V_{\lambda, k}\left(\kappa, \frac{1}{\lambda}\right)+\frac{\lambda^{2}-1}{2 \lambda^{3} \kappa} \cdot \frac{\partial}{\partial y} V_{\lambda, k}\left(\kappa, \frac{1}{\lambda}\right) \\
& =\frac{\partial}{\partial x} V_{\lambda, k}\left(\kappa, \frac{1}{\lambda}\right) \leq 0
\end{aligned}
$$


since the point $(\kappa, 1 / \lambda)$ is just on the curve defined by $\frac{\partial}{\partial y} V_{\lambda, k}=0$ and since $\eta(\kappa) \leq 1 / \lambda$; thus we have $\kappa \geq \delta_{\lambda, k}$. Therefore the maximum of $W_{\lambda, k}(x)$ for $\kappa \leq x<1$ is attained at $x=\kappa$; hence

$$
\max _{\kappa \leq x<1} W_{\lambda, k}(x)=W_{\lambda, k}(\kappa)=V_{\lambda, k}\left(\kappa, \frac{1}{\lambda}\right) .
$$

From (5.17) and (5.18), we obtain

$$
\max _{0<x<1} \max _{\sqrt{\nu} \leq y<1} V_{\lambda, k}(x, y)=F_{\lambda}\left(\beta_{\lambda, k}\right),
$$

which completes the proof of (1).

We next consider the case in which $\eta(\kappa) \geq 2 /(\lambda+1)=h_{+}(0)$. Then it is not difficult to see that this inequality is equivalent to condition (b). In this case the equations $h_{ \pm}(x)=\eta(x)$ have no positive solutions since $\kappa<\theta(\lambda)$ and $h_{+}(0) \leq \eta(\kappa)$. Thus

$$
\max _{0<x \leq \kappa} \max _{\sqrt{\nu} \leq y<1} V_{\lambda, k}(x, y)=V_{\lambda, k}\left(\kappa, \frac{1}{\lambda}\right)=W_{\lambda, k}(\kappa) .
$$

Moreover, $\kappa<\delta_{\lambda, k}$ since $W_{\lambda, k}^{\prime}(\kappa)=\frac{\partial}{\partial x} V_{\lambda, k}(\kappa, 1 / \lambda)>0$. This means that the maximum of $W_{\lambda, k}(x)$ is attained at $x=\delta_{\lambda, k} \in(\kappa, 1)$; therefore

$$
\max _{\kappa \leq x<1} W_{\lambda, k}(x)=W_{\lambda, k}\left(\delta_{\lambda, k}\right)=H_{\lambda}\left(\delta_{\lambda, k}\right) .
$$

From (5.19) and (5.20), we obtain

$$
\max _{0<x<1} \max _{\sqrt{\nu} \leq y<1} V_{\lambda, k}(x, y)=H_{\lambda}\left(\delta_{\lambda, k}\right),
$$

which completes the proof of (2).

Remark. The estimate (5.10) is also valid in any case in which the algebraic equation (5.1) has no positive roots. This follows from the fact that the function $V_{\lambda, k}\left(x, h_{+}(x)\right)$ is strictly increasing and $\kappa<\delta_{\lambda, k}$.

Finally, concerning the estimate of $I_{\lambda, N}(k)$, we have

$$
\limsup _{N \rightarrow \infty} \frac{1}{N} \log \left|I_{\lambda, N}(k)\right| \leq \limsup _{N \rightarrow \infty} \frac{1}{N} \log \left|J_{\lambda, N}(k)\right|
$$

for any integer $k \neq 0,1$, since

$$
I_{\lambda, N}(k)=-\int_{0}^{1} \frac{P_{\lambda, N}(t)}{k-t} d t=(-1)^{l+1} \Lambda_{N}(k) .
$$

\section{Main Results}

We are now ready to state our main theorem. For brevity, put

$$
\sigma \equiv \sigma(\lambda, k)=4 \lambda+2-e(\lambda)+F_{\lambda}\left(\alpha_{\lambda, k}\right) .
$$

We also put

$$
\tau \equiv \tau(\lambda, k)=e(\lambda)-4 \lambda-2-\max \left\{F_{\lambda}\left(\beta_{\lambda, k}\right), F_{\lambda}\left(\gamma_{\lambda, k}\right)\right\}
$$

for every positive integer $k \geq 2$ and

$$
\tau \equiv \tau(\lambda, k)=e(\lambda)-4 \lambda-2- \begin{cases}F_{\lambda}\left(\beta_{\lambda, k}\right) & \text { if } \kappa \geq \frac{\lambda^{2}+\lambda-1}{3 \lambda^{2}+\lambda-1}, \\ H_{\lambda}\left(\delta_{\lambda, k}\right) & \text { if } \kappa \leq \frac{2 \lambda^{2}+2 \lambda-1}{6 \lambda^{2}+4 \lambda-1},\end{cases}
$$

for every negative integer $k$. Then we have 
Theorem 6.1. Let $k \neq 0,1$ be an integer and let $\lambda>1$ be a real number satisfying $\tau \equiv \tau(\lambda, k)>0$. Then the number $L_{2}(1 / k)$ is irrational; more precisely, for any $\varepsilon>0$, there exists a positive integer $q(\varepsilon)$ such that

$$
\left|L_{2}\left(\frac{1}{k}\right)-\frac{p}{q}\right| \geq q^{-1-\sigma / \tau-\varepsilon}
$$

for any integer $q \geq q(\varepsilon)$ and for all $p \in \mathbf{Z}$. $\left(L_{2}(1 / k)\right.$ has an irrationality measure $1+\sigma / \tau$.)

Proof. Putting

$$
p_{N}=\frac{M_{N}^{2}}{d_{\lambda}(N)} P_{\lambda, N}(k), \quad q_{N}=\frac{M_{N}^{2}}{d_{\lambda}(N)} Q_{\lambda, N}(k), \quad \varepsilon_{N}=\frac{M_{N}^{2}}{d_{\lambda}(N)} J_{\lambda, N}(k),
$$

it follows from (4.2) that

$$
p_{N} L_{2}\left(\frac{1}{k}\right)-q_{N}=\varepsilon_{N}
$$

Clearly $p_{N}$ and $q_{N}$ are some integers for all $N \geq 0$. Note that $p_{N} \neq 0$ for all $N \geq 0$ by Lemma 2.2. Thus $q_{N} / p_{N}$ is the $N$ th approximate rational number to $L_{2}(1 / k)$ and $\varepsilon_{N}$ is the remainder term. Then, by Lemmas 3.1, 5.1, 5.2, and 5.3 , it is easily seen that

$$
\limsup _{N \rightarrow \infty} \frac{1}{N} \log \left|p_{N}\right| \leq \frac{\sigma(\lambda, k)}{2 \lambda+1}
$$

and

$$
\limsup _{N \rightarrow \infty} \frac{1}{N} \log \left|\varepsilon_{N}\right| \leq-\frac{\tau(\lambda, k)}{2 \lambda+1} .
$$

Let $V_{N}$ be the set of the $K+1$ points

$$
\frac{q_{N}}{p_{N}}, \frac{q_{N+1}}{p_{N+1}}, \ldots, \frac{q_{N+K}}{p_{N+K}}
$$

for all $N \geq 0$. Suppose now that $V_{M}$ consists of a single point for some $M$. Then, from (6.1),

$$
\frac{\varepsilon_{M}}{p_{M}}=\frac{\varepsilon_{M+1}}{p_{M+1}}=\cdots=\frac{\varepsilon_{M+K}}{p_{M+K}}, \quad \text { say } \mu ;
$$

that is, $\mu P_{\lambda, j}(k)-J_{\lambda, j}(k)=0$ for $M \leq j \leq M+K$. Thus, by Corollary 2.4, this holds for all $j \geq M$; hence $\varepsilon_{j} / p_{j}=\mu$ for all $j \geq M$. On the other hand, since $\left|p_{j}\right| \geq 1$ and $\tau(\lambda, k)>0$, we have

$$
\left|\frac{\varepsilon_{j}}{p_{j}}\right| \leq\left|\varepsilon_{j}\right| \rightarrow 0 \text { as } j \rightarrow \infty ;
$$

thus $\mu=0$. This implies that $J_{\lambda, j}(k)=0$ for all $j \geq M$, contrary to Corollary 2.6. Hence the set $V_{N}$ contains at least two distinct points for any $N \geq 0$. Therefore $L_{2}(1 / k)$ has an irrationality measure $1+\sigma / \tau$ by Lemma 3.2 in [7, p. 108]. This completes the proof.

Proof of Theorem 1.1. Using the above theorem we can give the proof of Theorem 1.1 as follows. We first show that $\tau(3, k)>0$ for every integer $k \geq 7$. From equation (5.1) we find that $\left\{\beta_{3, k}\right\}_{k \geq 7} \subset\left(\frac{1}{4}, \frac{1}{3}\right)$ is a strictly increasing sequence satisfying $\beta_{3, k} \rightarrow \frac{1}{3}$ as $k \rightarrow \infty$, and $\left\{\gamma_{3, k}\right\}_{k \geq 7} \subset\left(\frac{5}{11}, \frac{1}{2}\right)$ is a strictly 
decreasing sequence satisfying $\gamma_{3, k} \rightarrow \frac{5}{11}$ as $k \rightarrow \infty$. Thus both $\left\{F_{3}\left(\beta_{3, k}\right)\right\}$ and $\left\{F_{3}\left(\gamma_{3, k}\right)\right\}$ are strictly decreasing sequences; hence $\tau(3, k) \geq \tau(3,7)$ for every $k \geq 7$. Now it is easily seen that $0.327<\beta_{3,7}<\gamma_{3,7}<0.481$, $F_{3}(0.327)<-12$, and $F_{3}(0.481)<-12$; therefore, from (3.6), we have

$$
\tau(3,7)=e(3)-14-\max \left\{F_{3}\left(\beta_{3,7}\right), F_{3}\left(\gamma_{3,7}\right)\right\}>e(3)-2>0,
$$

as required.

We next show that $\tau(4, k)>0$ for every integer $k \leq-5$. Condition (a) is clearly fulfilled by $\lambda=4$ and every $k \leq-6$. Moreover, $\left\{\beta_{4, k}\right\}_{k \leq-6}$ is a strictly decreasing sequence satisfying $\beta_{4, k} \rightarrow \frac{1}{3}$ as $k \rightarrow-\infty$. Hence $\left\{F_{4}\left(\beta_{4, k}\right)\right\}_{k \leq-6}$ is also a strictly decreasing sequence; thus $\tau(4, k) \geq \tau(4,-6)$ for every $k \leq$ -6 . Since $\beta_{4,-6}<0.36$ and $F_{4}(0.36)<-15.5$, we have, from (3.7),

$$
\tau(4,-6)=e(4)-18-F_{4}\left(\beta_{4,-6}\right)>e(4)-2.5>0 \text {. }
$$

Finally, since condition (b) is fulfilled by $\lambda=4$ and $k=-5$, we have

$$
\tau(4,-5)=e(4)-18-H_{4}\left(\delta_{4,-5}\right) \quad \text { with } \delta_{4,-5}=\frac{\sqrt{1249}-32}{9} .
$$

Now it is easily seen that $H_{4}\left(\delta_{4,-5}\right)<-15.3$; hence $\tau(4,-5)>e(4)-2.7>0$. This completes the proof of Theorem 1.1.

In general it seems to be difficult to find the exact parameter $\lambda^{*}(k)$ at which the irrationality measure

$$
\mu(\lambda, k)=1+\frac{\sigma(\lambda, k)}{\tau(\lambda, k)}
$$

attains its minimum as $\lambda \in(1, \infty)$ varies. Moreover, in several cases, it seems that $\lambda^{*}(k)$ is not an integer. This will be a new phenomenon because the best parameters were certainly integers in the previous study [7] on the Padé-type approximations to the numbers such as $\log 2, \pi / \sqrt{3}, \pi^{2}$, and $\zeta(3)$. Some numerical examples on the irrationality measures $\mu(\lambda, k)$ of the numbers $L_{2}(1 / k)$ derived from our main theorem are given on the next page in Tables 1 and 2 . Here each parameter $\lambda(k)$ is taken to be a rational number with relatively small denominator in order to calculate the exponent $e(\lambda)$ exactly using Lemma 3.3. In each case there may be small room for improvement by choosing more complicated rational parameters. Indeed, for each fixed integer $k \geq 7$, the parameter $\lambda(k)$ is chosen so that $\lambda(k)$ is an approximate rational number to a (presumably unique) solution $\tilde{\lambda}(k)$ of the equation $F_{\lambda}\left(\beta_{\lambda, k}\right)=F_{\lambda}\left(\gamma_{\lambda, k}\right)$. For example, by taking $\lambda=\frac{433}{158}$, one will find that

$$
\left|L_{2}\left(\frac{1}{7}\right)-\frac{p}{q}\right| \geq q^{-95.0002}
$$

for any sufficiently large integer $q$.

Corollary 2.6 enables us to show the linear independence over $\mathbf{Q}$ of the numbers $1, \log (1-1 / k)$, and $L_{2}(1 / k)$ for every integer $k \in(-\infty,-5] \cup$ $[7, \infty)$. To see this, suppose that

$$
u_{0}+v_{0} \log \left(1-\frac{1}{k}\right)+w_{0} L_{2}\left(\frac{1}{k}\right)=0
$$


TABLE 1. ( $k:$ positive $)$

\begin{tabular}{rccr}
$k$ & $\begin{array}{c}\text { parameters } \\
k(k)\end{array}$ & $\begin{array}{c}\text { exponents } \\
e(\lambda)\end{array}$ & $\begin{array}{c}\text { irrationality } \\
\text { measures } \mu(\lambda, k)\end{array}$ \\
\hline 7 & $74 / 27$ & $2.05302 \ldots$ & $95.0605 \ldots$ \\
8 & $56 / 19$ & $2.05001 \ldots$ & $51.0848 \ldots$ \\
9 & $22 / 7$ & $2.17576 \ldots$ & $33.1311 \ldots$ \\
10 & $10 / 3$ & $2.32601 \ldots$ & $25.2396 \ldots$ \\
11 & $7 / 2$ & $2.43808 \ldots$ & $21.1863 \ldots$ \\
12 & $11 / 3$ & $2.55718 \ldots$ & $18.5439 \ldots$ \\
13 & $23 / 6$ & $2.68159 \ldots$ & $16.6546 \ldots$ \\
14 & 4 & $2.81244 \ldots$ & $15.2749 \ldots$ \\
15 & $29 / 7$ & $2.84624 \ldots$ & $14.4767 \ldots$ \\
16 & $30 / 7$ & $2.88745 \ldots$ & $13.8057 \ldots$ \\
17 & $31 / 7$ & $2.93273 \ldots$ & $13.2385 \ldots$ \\
18 & $73 / 16$ & $2.97250 \ldots$ & $12.7562 \ldots$
\end{tabular}

TABLE 2. ( $k:$ negative)

\begin{tabular}{ccccc}
\multicolumn{3}{c}{ parameters } & exponents & $\begin{array}{c}\text { irrationality } \\
k\end{array}$ \\
$\lambda(k)$ & case & $e(\lambda)$ & measures $\mu(\lambda, k)$ \\
\hline-5 & 4 & (b) & $2.81244 \ldots$ & $228.612 \ldots$ \\
-6 & 4 & (a) & $2.81244 \ldots$ & $55.9375 \ldots$ \\
-7 & $22 / 5$ & (a) & $2.92437 \ldots$ & $35.8351 \ldots$ \\
-8 & 6 & (b) & $3.54658 \ldots$ & $27.3905 \ldots$ \\
-9 & 6 & (b) & $3.54658 \ldots$ & $22.6583 \ldots$ \\
-10 & 6 & (b) & $3.54658 \ldots$ & $19.7479 \ldots$ \\
-11 & 6 & (b) & $3.54658 \ldots$ & $17.7685 \ldots$ \\
-12 & 6 & (b) & $3.54658 \ldots$ & $16.3297 \ldots$ \\
-13 & 6 & (a) & $3.54658 \ldots$ & $15.2424 \ldots$ \\
-14 & $67 / 11$ & (a) & $3.57289 \ldots$ & $14.4179 \ldots$ \\
-15 & $25 / 4$ & (a) & $3.61686 \ldots$ & $13.7503 \ldots$ \\
-16 & $32 / 5$ & (a) & $3.65638 \ldots$ & $13.1953 \ldots$
\end{tabular}

for some integers $u_{0}, v_{0}$, and $w_{0}$. Then, from (4.2) and (4.4), we have

$$
u_{0} p_{N}+v_{0} r_{N}+w_{0} q_{N}+v_{0} \eta_{N}+w_{0} \varepsilon_{N}=0
$$

for all $N \geq 0$, where

$$
r_{N}=\frac{M_{N}^{2}}{d_{\lambda, N}} R_{\lambda, N}(k) \quad \text { and } \quad \eta_{N}=\frac{M_{N}^{2}}{d_{\lambda, N}} I_{\lambda, N}(k) .
$$

Since there exists a $\lambda$ satisfying $\tau(\lambda, k)>0$ for each fixed $k \in(-\infty,-5] \cup$ $[7, \infty)$, we have $\eta_{N} \rightarrow 0$ and $\varepsilon_{N} \rightarrow 0$ as $N \rightarrow \infty$ from (5.21). Hence $v_{0} \eta_{N}+w_{0} \varepsilon_{N}=0$ for all sufficiently large $N$ since $u_{0} p_{N}+v_{0} r_{N}+w_{0} q_{N} \in \mathbf{Z}$; thus $v_{0}=w_{0}=0$ by Corollary 2.6 , as required.

Moreover, analogously to [8], we can obtain the measure of the linear independence, as follows. 
Theorem 6.2. If $\tau(\lambda, k)>0$, then, for any $\varepsilon>0$, there exists a positive integer $H(\varepsilon) \equiv H(\lambda, k ; \varepsilon)$ such that

$$
\left|p+q \log \left(1-\frac{1}{k}\right)+r L_{2}\left(\frac{1}{k}\right)\right| \geq H^{-\sigma / \tau-\varepsilon}
$$

for any integers $p, q$, and $r$ with $H \equiv \max \{|q|,|r|\} \geq H(\varepsilon)$.

This follows easily from the following property: Let $u_{0}, v_{0}$, and $w_{0}$ be arbitrary integers with $\left(u_{0}, v_{0}, w_{0}\right) \neq(0,0,0)$. Then we have

$$
\max _{N \leq j \leq N+K}\left|u_{0} p_{j}+v_{0} r_{j}+w_{0} q_{j}\right| \geq 1
$$

for any integer $N \geq 0$.

\section{REFERENCES}

1. F. Beukers, $A$ note on the irrationality of $\zeta(2)$ and $\zeta(3)$, Bull. London Math. Soc. 11 (1979), 268-272.

2. G. V. Chudnovsky, Padé approximations to the generalized hypergeometric functions. I, J. Math. Pures Appl. 58 (1979), 445-476.

3. __ Measures of irrationality, transcendence and algebraic independence. Recent progress (Number Theory Days, 1980), London Math. Soc. Lecture Note Ser., vol. 56, Cambridge Univ. Press, Cambridge-New York, 1982, pp. 11-82.

4. D. V. Chudnovsky and G. V. Chudnovsky, Padé and rational approximations to systems of functions and their arithmetic applications, Lecture Notes in Math., vol. 1052, SpringerVerlag, 1984, pp. 37-84.

5. R. Dvornicich and C. Viola, Some remarks on Beukers' integrals (Number Theory, Budapest), Colloq. Math. Soc. János Bolyai, vol. 51, North-Holland, 1987, pp. 637-657.

6. A. Erdélyi et al., Higher transcendental functions, vol. 1, McGraw-Hill, New York, 1953.

7. M. Hata, Legendre type polynomials and irrationality measures, J. Reine Angew. Math. 407 (1990), 99-125.

8. __ On the linear independence of the values of polylogarithmic functions, J. Math. Pures Appl. 69 (1990), 133-173.

9. L. Lewin, Polylogarithms and associated functions, North-Holland, New York, 1981.

10. W. Maier, Potenzreihen irrationalen Grenzwertes, J. Reine Angew. Math. 156 (1927), 93148.

11. A. van der Poorten, A proof that Euler missed-Apéry's proof of the irrationality of $\zeta(3)$, Math. Intelligencer 1 (1979), 195-203.

12. E. A. Rukhadze, $A$ lower bound for the approximation of $\ln 2$ by rational numbers, Vestnik Moskov. Univ. Ser. I Math. Mekh. (1987), no. 6, 25-29. (Russian)

Institute of Mathematics, Yoshida College, Kyoto University, Kyoto 606, Japan 